\title{
Spouse Distress Predicts Health Outcomes in Patients with Heart Failure
}

\author{
Audrey Cleary, Michael J. Rohrbaugh, Varda Shoham, \& Emily A. Butler \\ University of Arizona
}

Western Psychological Association, Palm Springs, CA, April 2006

\begin{abstract}
Psychological distress reported by the spouses of 60 heart failure patients predicted changes in the patients' physical health over the next six months independent of baseline illness severity and the patient's own distress. Negative spouse emotion also had a marginally significant effect on patient survival.

\section{Background}

Chronic heart disease is a potential source of psychological distress for both patients and their spouses. While research suggests that negative emotions such as depression, anxiety, and hostility influence the course of a patient's own illness, less is known about health consequences of the spouse's distress for either the spouse him/herself or for the patient.

In a recent cross-sectional study of couples coping with congestive heart failure (CHF), we found high levels of distress reported by patients and spouses, as well as gender differences in spouse distress associated with the quality of the couple's relationship (Rohrbaugh et al., 2002). Related studies with the same (Michigan) sample further implicated marital quality as a factor in patient survival (Coyne et al., 2001; Rohrbaugh et al., 2004).

The present sample used a different sample of CHF couples to examine health consequences of patient and spouse distress prospectively, both within and between partners. Analyses focused on whether each partner's psychological distress and general positive/negative emotions predicted future declines in the other partner's physical health, over and above what distress/emotion in the actor could predict (Kenny, 1996). Of particular interest was whether the spouse's distress affected the patient's health more than vice versa.
\end{abstract}

\section{Method}

Participants were $59 \mathrm{CHF}$ patients and their spouses recruited from University of Arizona cardiology clinics. Mean patient age was 64.3 years, and mean CHF severity (New York Heart Association class) was 2.3 on a $1-4$ scale. Patients were $72 \%$ male, $85 \%$ white and $42 \%$ college educated. About half $(53 \%)$ experienced at least minor adverse health change during the follow-up period, and 12 died within 3 years of the assessment.

In separate home interviews, patients and spouses completed (a) the Hopkins Symptom Checklist-25 (HSCL-25; Heshbacher et al., 1978), a measure of psychological distress, (b) the Positive and Negative Affect Scale (PANAS), a non-clinical measure of emotional experience; and (c) the SF-36 Health Questionnaire (McHorney et al., 1993), a widely used self-report measure of health. Participants answered the SF-36 questions again in a telephone follow-up interview 6 months later. Following Barefoot et al. (2000), we combined the SF-36 physical functioning, bodily pain, and vitality subscales into a composite measure of physical health to minimize overlap of the dependent variable with the predictor constructs of distress and positive/negative emotion. We also monitored all-cause mortality over the next three years and confirmed death dates from public records.

\section{Results}

In preliminary analyses, HSCL and PANAS negative affect scores correlated .66 for patients and .76 for spouses, but both were relatively independent of reported positive emotion. HSCL scores were in the clinical (distressed) range for $37 \%$ of patients and $20 \%$ of spouses. Mixed-model ANOVAs revealed no gender differences, but patients reported more distress $(p<.01)$ and less positive affect $(p<.01)$ than spouses, and CHF severity was more associated with patient distress than with spouse distress (role $\mathrm{x}$ severity interaction, $p=.05$ ). 
Multiple regression analysis comparing patient and spouse characteristics as predictors of six-month residualized change in the patient's physical health, with baseline illness severity and the patient's emotion score controlled, found significant spouse (partner) effects for HSCL distress (standardized $b=$ $.23, p=.02)$ and PANAS negative affect $(b=-.25, p=.05)$. In other words, the spouse's negative emotions appeared to contribute independently to the patient's perceived health, over and above what the patient's own distress could predict. The same regression models also revealed significant actor effects for the patient's baseline distress $(b=-.25, p=.02)$ and positive affect $(b=.20, p=.03)$. By contrast, neither actor (patient) nor partner (spouse) effects were evident when outcomes concerned the spouse's health rather than the patient's.

With death events aggregated by month, Cox proportional-hazards regression also showed a nearsignificant partner effect of the spouse's negative affect on patient survival during the 3-year follow-up period ( $W$ ald $=3.43, p=.06$ ), although neither actor nor partner effects on survival reached significance for the HSCL or positive affect measures.

\section{Conclusions}

The results add to growing evidence that close relationships are important to physical health. In particular, statistical partner effects suggest that a spouse's negative affectivity can influence the course of congestive heart failure (and perhaps even the patient's survival) over and above the patient's own distress. These partner effects were asymmetrical, however, in that the patient's distress did not appear to influence the spouse's health in a similar fashion. Finally, although positive emotions on the part of the patient did appear to have short-term health benefits (an actor effect), the spouse's contribution was entirely in the realm of negative emotion.

More generally, the results highlight the importance of looking beyond the patient in health psychology research, as data on spouses' psychological functioning can improve prediction of patient outcomes.

\section{References}

Barefoot, J.C., Brummett, B.H., Clapp-Channing, N.E., Siegler, I.C., Vitaliano, P.P., Williams, R.B., \& Mark, D.B. (2000). Moderators of the effect of social support on depressive symptoms in cardiac patients. American Journal of Cardiology, 86, 438-442.

Coyne, J.C., Rohrbaugh, M.J., Shoham, V., Cranford, J.A.,, Nicklas, J.M., \& Sonnega, J. (2001). Prognostic importance of marital quality for survival of congestive heart failure. American Journal of Cardiology, 88, 526-529.

Heshbacher, P.K., Downing, R.W., \& Stephansky, P. (1978). Assessment of psychiatric illness severity by family physicians. Social Science and Medicine, 12, 45-52.

Kenny, D.A. (1996). The design and analysis of social-interaction research. Annual Review of Psychology, 47, 59-86.

McHorney C, Ware J, et al. (1993). The MOS-Item Short-Form Health Survey (SF-36). Medical Care, 31, 247-63.

Rohrbaugh, M.J., Cranford, J.A., Shoham, V., Nicklas, J.M., Sonnega, J. \& Coyne, J.C. (2002). Couples coping with congestive heart failure: Role and gender differences in psychological distress. Journal of Family Psychology, 16, 3-13.

Rohrbaugh, M.J., Shoham, V., Coyne, J.C., Cranford, J.A.,, Nicklas, J.M., \& Sonnega, J. (2004). Beyond the "self" in self-efficacy: Spouse confidence predicts survival following heart failure. Journal of Family Psychology, 18, 184-193.

Correspondence address: Audrey Cleary, Department of Psychology, University of Arizona, P.O. Box 210068, Tucson, AZ 85721-0068 (e-mail: acleary@email.arizona.edu). 